\title{
Ataxia associated with Hashimoto's disease: progressive non-familial adult onset cerebellar degeneration with autoimmune thyroiditis
}

\author{
M Selim, D A Drachman
}

\begin{abstract}
Acquired cerebellar ataxia has been described with hypothyroidism, and is typically reversible by thyroid hormone replacement therapy. The cerebellar dysfunction has been attributed to metabolic and physiological effects of the endocrine disorder. In a few patients, however, ataxia has persisted despite thyroid replacement therapy. Other mechanisms may be involved in ataxia associated with thyroid disorders.
\end{abstract}

Objective-To document progressive nonfamilial adult onset cerebellar degeneration (PNACD) occurring in six patients with raised antithyroid antibodies (Hashimoto's/autoimmune thyroiditis), and other autoimmune manifestations, in the absence of hypothyroidism; and to document the independence of the cerebellar disorder from the endocrine dysfunction.

Methods-A case study of six patients with PNACD reviewing the clinical course and relation to endocrine and autoimmune status.

Results-All six patients were euthyroid when they developed their symptoms; had raised antithyroid antibodies consistent with Hashimoto's autoimmune thyroiditis; and had strong personal or family histories of organ specific autoimmune diatheses. Brain MRI disclosed atrophy of the cerebellar vermis in four patients and olivopontocerebellar atrophy in two. Other possible causes of cerebellar degeneration were excluded. De novo treatment (two patients) or continued treatment (three patients) with L-thyroxine did not modify the progression of the ataxia.

Conclusions-Cerebellar degeneration in these patients with raised antithyroid antibodies may be immune mediated. The presence of antithyroid antibodies may signal or cause the autoimmune process producing cerebellar degeneration.

Massachusetts Medical School, 55 Lake Ave North, Worcester, MA 01655, USA

M Selim

D A Drachman

Correspondence to: Dr D A Drachman david.drachman@ umassmed.edu

Received 30 October 2000 and in revised form

9 February 2001

Accepted 16 February 2001 represent a recognisable and not uncommon condition; a trial of immunomodulating therapy should be considered in these patients.

(f Neurol Neurosurg Psychiatry 2001;71:81-87)

Keywords: ataxia; autoimmune; cerebellar degeneration; Hashimoto's autoimmune thyroiditis

Some conditions are well recognised to produce progressive non-familial adult onset cerebellar degeneration (PNACD), including nutritional deficiencies, ${ }^{1}$ intoxication, ${ }^{2}$ hypoxia, hyperthermia, paraneoplastic syndromes, ${ }^{3}$ and olivopontocerebellar degeneration of unknown cause, described by Déjerine and Thomas. ${ }^{4}$ Hypothyroidism has been described as a cause of gait ataxia, presumably due to cerebellar dysfunction, ${ }^{5-15}$ although the exact mechanism(s) by which it might produce this syndrome is unclear. In most reported cases, the ataxia has been reversed by thyroid replacement therapy, ${ }^{7-12}$ suggesting that it was caused by the metabolic and physiological effects of the hormonal deficiency. ${ }^{711} 1214$ In some patients, however, despite thyroid replacement therapy the cerebellar syndrome has persisted and progressed..$^{5-7111516}$

In this paper we report on six patients, seen at our institution between 1987 and 1999, presenting with PNACD and raised antithyroid antibody titres diagnostic of Hashimoto's autoimmune thyroiditis (table 1) ${ }^{17}$ All the patients were euthyroid at the time of presentation, and their cerebellar symptoms progressed despite remaining euthyroid, with maintenance of adequate thyroid replacement therapy as needed. Infectious, paraneoplastic, vascular, metabolic, nutritional, and alcohol related causes of cerebellar degeneration were excluded. All six patients had a strong personal or family history of additional autoimmune disorders, suggesting that autoimmunity associated with Hashimoto's autoimmune thyroiditis may produce a syndrome of progressive cerebellar degeneration.

Table 1 Cerebellar symptoms and associated deficits at presentation and during subsequent course.

\begin{tabular}{|c|c|c|c|c|c|c|}
\hline Symptom & Patient 1 & Patient 2 & Patient 3 & Patient 4 & Patient 5 & Patient 6 \\
\hline Gait ataxia & At onset & At onset & At onset & Subsequent & At onset & Subsequent \\
\hline Hand incoordination & Late & At onset & Late & Late & Late & At onset \\
\hline Dysarthria & Late & Late & Late & Late & Late & At onset \\
\hline Diplopia & Late & & & Late & At onset & \\
\hline Nystagmus & Late & & & & At onset & \\
\hline Vertigo & & & Late & & Late & \\
\hline Dizziness "unspecified" & At onset & & & & & \\
\hline Hearing impairment & & & & & At onset & At onset \\
\hline Peripheral neuropathy & At onset & & & & At onset & \\
\hline Extrapyramidal features & & & & At onset & & At onset \\
\hline
\end{tabular}




\section{Case histories}

CASE 1

A 57 year old woman was seen for evaluation of longstanding gait imbalance and lightheadedness when walking. Her symptoms began after an episode of ulcerative colitis, which culminated in total colectomy. Her medical history was indicative for rheumatoid arthritis. She rarely consumed alcohol. Both her mother and her daughter were known to have "thyroid disease." Her daughter also had autoimmune haemolytic anaemia. There was no family history of cerebellar, gait, or other neurological disorders in either parent or five brothers.

The patient had a palpable thyroid gland. Neurological examination disclosed a normal mental status. She had downbeat nystagmus, most marked on left lateral gaze, and impaired vestibulo-ocular and pursuit image stabilisation. Her pupils were slightly irregular and responded sluggishly to light. Her gait was broad based and unsteady; her Romberg test was mildly impaired, and she was unable to walk tandem. Strength and tone were normal in all limbs. Deep tendon reflexes were normal in the upper limbs, reduced at the knees, and absent at the ankles. The plantar reflexes were mute. All primary sensory modalities were diminished below the midtibial region. In the upper limbs, there was only a mild distal decrease of temperature and vibration sensation. Bárány rotation failed to produce postrotatory vertigo.

Brain MRI showed severe atrophy of the cerebellar vermis with enlargement of the adjacent fourth ventricle. The cerebellar hemispheres were spared and the olives were normal in size. The patient was euthyroid with a normal TSH, total T4, free thyroxine index (FTI), and T3 resin uptake (table 2 ). Both thyroglobulin antibody (TG-Ab) and thyroperoxidase antibody (TPO-Ab) were raised at $2.9 \mathrm{IU} / \mathrm{ml}$ and $>30$ $\mathrm{IU} / \mathrm{ml}$, respectively, consistent with the diagnosis of Hashimoto's thyroiditis; a thyroid scan demonstrated a normal ${ }^{123} \mathrm{I}$ uptake of $17 \%$ at 6 hours. Antinuclear antibody (ANA) was positive with a diffuse pattern and 1:160 titre; erythrocyte sedimentation rate (ESR) was raised at $50 \mathrm{~mm} /$ hour; and serum protein electrophoresis (SPEP) showed a slight increase in IgM without monoclonal spikes.

Investigation for malignancy was unrevealing. AntiPurkinje cell antibodies were not detected in the serum. Heavy metal screen, vitamin $\mathrm{B} 12$, vitamin $\mathrm{E}$, vitamin $\mathrm{B} 6$, rheumatoid factor, fluorescent treponemal antibody absorption test (FTA-Abs), Lyme serology, and CSF analysis were all normal.

Table 2 Results of thyroid function tests at presentation.

\begin{tabular}{llcrl}
\hline Patient & $\begin{array}{l}\text { TSH }(0.3-6 \\
\mu u / m l)\end{array}$ & $\begin{array}{l}\text { T4 (total) } \\
(5-12 \mu g / d l)\end{array}$ & \multicolumn{1}{c}{ TG-Ab } & TPO-Ab \\
\hline 1 & 1.81 & 8.4 & $2.9(0.3-1 \mathrm{IU} / \mathrm{ml})$ & $>30(0.3-1 \mathrm{IU} / \mathrm{ml})$ \\
2 & 0.42 & 10.2 & $1.7(0.3-1 \mathrm{IU} / \mathrm{ml})$ & $13.2(0.3-1 \mathrm{IU} / \mathrm{ml})$ \\
3 & 0.52 & 10.4 & $16.9(0.3-1 \mathrm{IU} / \mathrm{ml})$ & $22.1(0.3-1 \mathrm{IU} / \mathrm{ml})$ \\
4 & 0.45 & 9.6 & $1.8(0.3-1 \mathrm{IU} / \mathrm{ml})$ & $3.7(0.3-1 \mathrm{IU} / \mathrm{ml})$ \\
5 & 0.86 & 6.2 & $1.5(0.3-1 \mathrm{IU} / \mathrm{ml})$ & $2(0.3-1 \mathrm{IU} / \mathrm{ml})$ \\
6 & 3.87 & 6.5 & $<20(<35 \mathrm{IU} / \mathrm{ml})$ & $82.7(<35 \mathrm{IU} / \mathrm{ml})$ \\
\hline TSH=Thyroid & stimulating & hormone; & T4=thyroxine; TG-Ab=thyroglobulin antibody,
\end{tabular}

$\mathrm{TPO}-\mathrm{Ab}=$ thyroperoxidase antibody; normal values are given in parentheses.
The patient was treated with L-thyroxine, but continued to deteriorate despite a normal thyroid profile on repeated occasions. Six years after initial evaluation she was able to ambulate only with the aid of a walker and had developed mild scanning dysarthria, dysmetria on fingernose and heel-shin testing, exaggerated rebound, and loss of the check reflexes. Her intellect and cognition remained unaffected.

\section{CASE 2}

A 35 year old white woman presented with progressive gait ataxia of 5 years' duration. She noted clumsiness of her hands with difficulty writing, tying shoelaces, and pouring from a teapot. Her medical history was unremarkable and alcohol consumption was minimal. Her mother had diabetes mellitus and a goitre. Her father had died from bladder cancer. Her siblings were healthy with no known neurological disorders.

General physical examination was normal. Neurological examination showed scanning dysarthria, minimal slowing of extraocular movements on rapid saccades, loss of the check reflex in the upper limbs, slight dysmetria and slowing of rapid alternating movements in the upper limbs, and marked bilateral dysmetria on heel tapping. Her gait and stance were wide based and ataxic, and she was unable to walk tandem. The Romberg test was mildly impaired. The remainder of the neurological examination was normal.

Brain MRI disclosed profound atrophy of the midline cerebellum, with enlargement of the fourth ventricle. The pons and other brainstem structures appeared normal (fig $1 \mathrm{~A}$ ). Her $\mathrm{TSH}$ and T4 were normal; both TG-Ab and $\mathrm{TPO}-\mathrm{Ab}$ were increased at $1.7 \mathrm{IU} / \mathrm{ml}$ and 13.2 $\mathrm{IU} / \mathrm{ml}$, respectively (table 2). Her ESR was 38 $\mathrm{mm} /$ hour. Serum protein electrophoresis showed a slight polyclonal increase in the gamma region.

Serum vitamin B12 concentration, rapid plasma reagin test (RPR), and Lyme serology were unremarkable. Analysis of CSF was normal. Anti-Hu, anti-Yo, and anti-Ri antibodies were not detected in either serum or CSF.

Hashimoto's autoimmune thyroiditis was diagnosed, and therapy with L-thyroxine was started without significant improvement. Three years later, she had left her job and moved to a single floor house because of increasing gait disability.

\section{CASE 3}

A 56 year old woman was evaluated for unsteadiness. Her symptoms had begun 6 years earlier with poor balance and difficulty going downstairs. Subsequently she had noted the gradual development of dysarthria, impaired handwriting, and worsening gait. She also complained of motion sickness and intermittent vertigo. She had been treated in the past with levodopa without significant benefit. Her disability accelerated and she had become unable to carry out activities of daily living. Her medical history showed hypothyroidism (treated with L-thyroxine, euthyroid), and hypertension. She did not consume alcohol. 

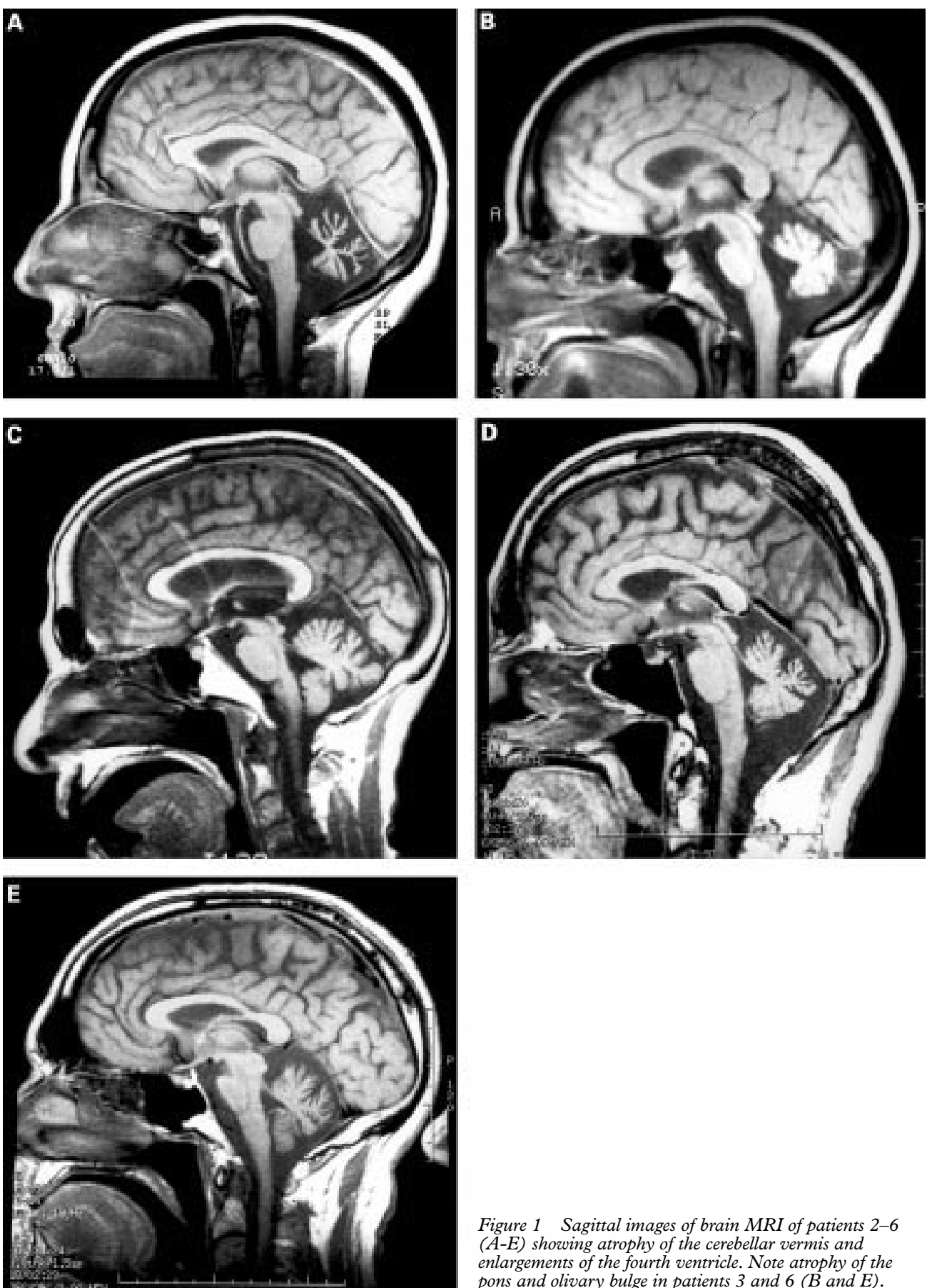

Figure 1 Sagittal images of brain MRI of patients 2-6 $(A-E)$ showing atrophy of the cerebellar vermis and enlargements of the fourth ventricle. Note atrophy of the pons and olivary bulge in patients 3 and 6 (B and $E$ ).

The patient's mother had been treated with radioactive iodine for hyperthyroidism, two maternal siblings had thyroid disease, and two of the patient's cousins also had a thyroid disorder. She had two children who were in good health; there was no family history of similar neurological symptoms.

Her general physical examination was unremarkable. The patient was of normal intelligence, and language was intact, but articulation was mildly dysarthric. Extraocular movements were saccadic on pursuit, and targeting disclosed undershoot. Gait and stance were broad based and ataxic, and tandem walking was impossible. Minimal truncal titubation was noted. Rapid alternating movements were slow

and clumsy. Heel-shin and finger-nose testing were dysmetric, and loss of the check reflex was noted. Muscle tone and power were normal. Deep tendon reflexes and primary sensory modalities were intact throughout.

Brain MRI showed generalised volume loss in the cerebellar hemispheres with enlargement of the fourth ventricle. Absence of the normal olivary bulge was also noted (fig $1 \mathrm{~B}$ ). Her thyroid profile showed normal concentrations of TSH, T4, T3 resin uptake, and FTI (table 2). Both TPO-Ab and TG-Ab were raised at 22.1 $\mathrm{IU} / \mathrm{ml}$ and $16.9 \mathrm{IU} / \mathrm{ml}$, respectively. Serum protein electrophoresis and immunofixation showed the presence of IgG $\kappa$ monoclonal pro- 
tein. The ESR was mildly increased at 31 $\mathrm{mm} /$ hour.

The patient was started on a therapeutic trial of prednisone for about 6 weeks without a significant beneficial effect; it was stopped because of exacerbation of her hypertension. Her speech and gait continued to deteriorate and she became wheelchair bound.

CASE 4

A 69 year old woman was referred for neurological evaluation because of slight slowing of her thinking and behaviour. The patient herself denied significant changes in her memory or thought process and was able to drive, travel, maintain two homes independently, and take care of her personal affairs. She had noticed slight impairment in her coordination while golfing, buttoning up clothes, and writing. Her medical history was indicative of rheumatoid arthritis, Raynaud's phenomenon, mitral valve prolapse, hypothyroidism (treated with L-thyroxine, euthyroid) and adult onset diabetes mellitus. She drank alcohol occasionally. Her father and twin brother had died of Alzheimer's disease, and her younger sister was thought to have mild cognitive impairment; no relatives had a disorder similar to the patient's.

The patient was oriented, but her speech and responses were slow and there was mild impairment of memory storage, calculation, and fund of knowledge. She was able to draw, copy, and write accurately. The remainder of her neurological examination was remarkable only for an occasional missed step on tandem walking, slightly diminished arm swing when walking, a mildly stooped posture, and tremulousness of the outstretched hands.

Head CT showed prominent ventricles and cortical sulci, appropriate for her age. FTA-Abs and TSH were normal. Antithyroid antibodies TG-Ab and TPO-Ab were raised at $1.8 \mathrm{IU} / \mathrm{ml}$ and $3.7 \mathrm{IU} / \mathrm{ml}$, respectively. Rheumatoid factor was markedly increased at $3+$ with a titre of 1:10 240, and ANA was 2+ with a speckled pattern and a titre of $1: 10$.

Formal psychometric testing showed average intellectual performance with lapses in concentration, but did not support the diagnosis of Alzheimer's disease. An EEG was normal. Serum chemistry, blood counts, vitamin B12, folate, SPEP, and a malignancy investigation were unremarkable.

The patient was given a trial of levodopa/ carbidopa, without significant benefit. Her balance, gait, and writing continued to deteriorate over the ensuing 4 years and she experienced progressive dysarthria and diplopia. She developed marked apraxia of gaze; slow and saccadic extraocular movements; and mild hearing impairment. Eventually she became unable to walk unaided. Finger-nose and heel-shin testing were dysmetric, rapid alternating movements were slow and irregular, and there was loss of the check reflex. Repeated psychometric testing showed no further decline in her memory or other cognitive functions. Brain MRI showed marked cerebellar atrophy and dilation of the fourth ventricle (fig $1 \mathrm{C}$ ). The patient died at the age of 78 , but postmortem examination was not performed.

\section{CASE 5}

A 46 year old woman was admitted to hospital for complaints of diplopia, worsening dysarthria, and gait difficulties. Three months before admission, she had been in hospital for prolonged diarrhoea with subsequent metabolic acidosis, pancreatitis, and non-Q wave myocardial infarction. Subsequently she complained of unsteadiness of gait, vertigo, hearing impairment, and mild dysarthria. Her extensive medical history included acute rheumatic fever as a child, discoid lupus, Jaccoud's arthropathy, bipolar disorder, and Hashimoto's thyroiditis. She had grown up as a foster child and did not know her biological family. Alcohol consumption had been excessive, but she had been abstinent for the previous 6 years. Her medications included meclizine and L-thyroxine.

Neurologically, she was fully oriented. Language and memory were intact; she had scanning dysarthria. Horizontal nystagmus was present on lateral gaze bilaterally. Her gait and stance were broad based and staggering, tandem gait was unsteady, and she had dysmetria of all limbs. There was exaggerated rebound and loss of the check reflex. She had normal tone and muscle bulk with slight distal weakness (5-/5) in all limbs. Deep tendon reflexes were absent at the ankles. Plantar reflexes were flexor. Sensation to primary modalities was diminished distally. Vertigo was not elicitable on Bárány rotation. Brain MRI showed diffuse cerebellar atrophy and no evidence of demyelination (fig $1 \mathrm{D}$ ); MRA was normal. TSH, T4, FTI, and T3 were all within normal limits. Both TG-Ab and TPO-Ab were mildly increased at $1.5 \mathrm{IU} / \mathrm{ml}$ and $2 \mathrm{IU} / \mathrm{ml}$ (0.3-1), respectively (table 2); the modest increases in antithyroid antibodies were considered the result of longstanding autoimmune thyroid disease. ANA was positive with a titre of $1: 60$

Electromyography, nerve conduction studies, and somatosensory evoked potentials were all unremarkable. Vitamin E, vitamin B12, RPR, rheumatoid factor, SPEP, and ESR were normal. Analysis of CSF showed the presence of oligoclonal bands and a slightly increased total protein of $50 \mathrm{mg} / \mathrm{dl}$, with no other abnormalities. Genetic testing for spinocerebellar ataxia (SCA1-SCA7), was negative. A malignancy survey was unrevealing. Anti-Ri, Anti$\mathrm{Hu}$, and Anti-Yo antibodies were absent in serum and CSF. Serum glutamic acid decarboxylase antibody (anti-GAD) was 2.6 units/ml (normal <2.5).

The patient's gait progressively deteriorated. She became wheelchair bound during the next 8 months. A repeat MRI was unchanged. A therapeutic trial of IVIg ( $1 \mathrm{~g} / \mathrm{kg} /$ day for two consecutive days) was given; 2 months later the patient was able to ambulate with a walker, but the remainder of her neurological examination and other cerebellar deficits were unchanged. 
CASE 6

A 61 year old man was referred for evaluation of slowly progressive unsteadiness of gait of 4-5 years' duration. He had noted slowly progressive difficulties in walking, writing, driving within a lane, and speaking clearly. His medical history was notable only for a punctured left eardrum and mild concussion. He had had urinary frequency, diminished libido, and impotence for 4 years. He drank alcohol occasionally and was taking no medication. There was no family history of cerebellar symptoms. A general physical examination was unremarkable. Memory, cognition, and language were normal. Handwriting was mildly jerky and speech was mildly dysarthric. Hearing in the left ear was diminished to whispered numerals. Gait and stance were mildly broad based and tandem gait was slightly unsteady. Rapid alternating movements were mildly dysmetric. The remainder of the neurological examination was unremarkable. Brain MRI showed minimal decrease in the size of the cerebellar folia without other abnormalities. An electronystagmogram was normal, and audiometry showed mild to moderate mixed hearing loss, more pronounced in the left ear.

His gait and speech gradually worsened over the ensuing 3 years. He became mildly forgetful and fell on some occasions when turning. $\mathrm{He}$ developed orthostatic hypotension. Ocular movements showed mild undershoot on lateral gaze, saccadic lateral gaze, and microsquare wave jerks. Minimal dysarthria, loss of the check reflex on the left, bilateral Babinski signs, and slight axial rigidity and hypertonia of the right leg developed. TSH, T3 resin uptake, and $\mathrm{T} 4$ were all within normal limits. The TPO-Ab titre was raised at $82.7 \mathrm{IU} / \mathrm{ml}$; thyroglobulin antibody was normal (table 2). Antinuclear antibody was positive with a titre of $1: 160$. A repeat brain MRI showed significant atrophy of the cerebellum and pons consistent with olivopontocerebellar atrophy (fig $1 \mathrm{E}$ ).

Analysis of CSF, SPEP, Lyme serology, blood chemistry, and cell counts were all normal. Paraneoplastic antibodies were not detected in the serum. Serum anti-GAD antibody was 2.5 units $/ \mathrm{ml}$.

\section{Discussion}

We have investigated six patients presenting with progressive, non-familial adult onset cerebellar degeneration (PNACD) and increased antithyroid antibodies indicating Hashimoto's/autoimmune thyroiditis. At the onset of their neurological disorders all the patients were euthyroid (table 2); three had been treated with L-thyroxine for previously noted hypothyroidism. They remained euthyroid while their neurological disorders progressed. All had evidence of more generalised autoimmune disorders. Ataxia of gait was the presenting cerebellar symptom in five patients, with dysarthria occurring later (table 1). In addition, the clinical range variably included ocular motor, brain stem, extrapyramidal, and peripheral nerve abnormalities; and a picture similar to Déjerine-Thomas sporadic olivopon- tocerebellar atrophy. ${ }^{48}$ Brain MRI showed midline cerebellar atrophy in all patients, with additional olivary and pontine atrophy in two.

Progressive non-familial adult onset cerebellar degeneration may occur in association with other conditions, including paraneoplastic syndromes, ${ }^{3}{ }^{19-21}$ excessive alcohol use, ${ }^{2}$ lithium intoxication, ${ }^{22}$ and nutritional deficiency. ${ }^{1}$ All these disorders were excluded in our patients; and negative family histories in five excluded the Holmes type of familial cortical cerebellar degeneration. ${ }^{18}{ }^{23}$ In patient 5, where the family history was unavailable, genetic testing for SCA1-7 was negative; genetic testing for hereditary ataxia was not available at the time of initial evaluation in the other patients, although detailed family histories were negative.

Hypothyroidism has previously been recognised as a cause of gait ataxia as well as other symptoms of cerebellar dysfunction. ${ }^{5-14}$ The onset and progression of this syndrome has mostly been described in relation to laboratory evidence supportive of decreased thyroid function. This is by contrast with the patients reported here, who were all euthyroid at the onset of their neurological disorders. The pathogenesis of cerebellar dysfunction in patients with decreased thyroid function is uncertain. Restoring a euthyroid state with L-thyroxine has reversed the cerebellar symptoms in most patients, ${ }^{7-12}$ suggesting that their symptoms were due to endocrine mediated dysfunction of the cerebellum. ${ }^{72}{ }^{12}$ Physiological reduction of cardiac output, cerebral blood flow, and reduced oxygen and glucose consumption by cerebellar neurons has been suggested. ${ }^{71}{ }^{25}$ Adams et $a l^{18}$ favoured slowed muscle relaxation (pseudomyotonia) over cerebellar dysfunction as the cause of ataxia in hypothyroidism.

In some patients with hypothyroidism, however, the cerebellar deficits have persisted or progressed despite thyroid replacement therapy. ${ }^{67111516}$ The progression of cerebellar dysfunction despite normalised thyroid function suggests that in these patients the ataxia may have been due to a mechanism other than the hypothyroid state, as in our six patients.

We think that there may be two separate mechanisms resulting in cerebellar dysfunction in patients with thyroid disorders. In hypothyroidism not associated with antithyroid autoimmunity, the endocrine disorder may produce cerebellar dysfunction that can be reversed by thyroid replacement therapy. In our patients, and some others with Hashimoto's/ autoimmune thyroiditis not reversed by thyroid replacement therapy, autoimmune mediated cerebellar degeneration is a more likely mechanism. In the present series, the cerebellar degeneration was often associated with additional autoimmune disorders in the affected patients and their families; and seems to represent a separate clinical entity.

Five of our patients were women, in whom autoimmune disorders are known to be more common $^{26}{ }^{27}$; all had increased concentrations of antithyroid antibodies (TPO-Ab in particular); and other immunological abnormalities 
were common. The occurrence of cerebellar degeneration has been described in several well identified autoimmune disorders including paraneoplastic syndromes, ${ }^{3}{ }^{19-2128}$ systemic lupus erythromatosus, ${ }^{29}$ and late onset diabetes mellitus, ${ }^{16}$ supporting this possible mechanism in association with Hashimoto's/autoimmune thyroiditis.

Three necropsies of patients with hypothyroidism and cerebellar ataxia have been reported. ${ }^{5031}$ Barnard et $a \bar{l}$ described marked atrophy of the anterosuperior portion of the vermis with loss of Purkinje cells and gliosis of the ventral pons in a patient with Hashimoto's thyroiditis and cerebellar ataxia. Price and $\mathrm{Netsky}^{30}$ reported non-specific degenerative changes and "neural myxoedema bodies" in the cerebellum, and middle and superior peduncles, but they did not specify whether their patient had Hashimoto's thyroiditis.

Hashimoto's, or chronic lymphocytic, thyroiditis, is often a subclinical condition. ${ }^{17}{ }^{26} \mathrm{It}$ is characterised by lymphocytic infiltration of the thyroid gland and circulating antithyroid antibodies to thyroglobulin or thyroid peroxidase, which are present in $70 \%-95 \%$ of patients. ${ }^{17} 2632$ Antithyroid antibodies can also be found in 5\%-10\% of normal subjects ${ }^{26}{ }^{32}-\mathrm{a}$ figure which corresponds to the results at our institution, but differs from our experience in patients presenting with PNACD. A range of autoimmune diseases has been associated with Hashimoto's thyroiditis, including rheumatoid arthritis, systemic lupus erythromatosus, ulcerative colitis, pernicious anaemia, myasthenia gravis, multiple sclerosis, and thyroid ophthalmopathy. ${ }^{18}{ }^{27}$ 32-35 An encephalopathy has been reported in association with subclinical Hashimoto's thyroiditis, ${ }^{36-39}$ and is steroid responsive in some patients, ${ }^{36} 38$ indicating a likely autoimmune pathogenesis, and possibly an underlying cerebral vasculitis. ${ }^{37} 39$

Most patients with Hashimoto's thyroiditis do not develop an associated cerebellar disorder. Some $20 \%$ to $33 \%$ of patients with hypothyroidism may develop ataxia if not adequately treated. ${ }^{15}{ }^{40}$ The reason for the occurrence of cerebellar dysfunction in some, but not all, hypothyroid patients is not clear; additional metabolic and infectious factors, or surgical stress may contribute.

There are several mechanisms by which autoimmunity associated with Hashimoto's thyroiditis might induce cerebellar degeneration. As widespread autoimmune reactivity can be seen in patients with Hashimoto's disease, ${ }^{17}$ cerebellar degeneration may be mediated by another unidentified circulating antiPurkinje cell antibody. In this case, the autoimmune thyroiditis would be serving as a "marker," identifying the presence of a more generalised autoimmune disorder involving the cerebellum. Alternatively, if immunological cross reactivity exists between shared thyroid and cerebellar antigens, antithyroid antibodies could specifically affect the cerebellum. We are not aware of any substantial evidence implicating the presence of antibodies against cerebellar neurons in patients with Hashimoto's thyroiditis. One candidate might be antibodies to glutamic acid decarboxylase (GAD-Ab ${ }^{41}$. Abnormalities of this enzyme have been demonstrated in tissues from patients with olivopontocerebellar atrophy ${ }^{42}{ }^{43}$ and GAD antibodies have been detected in some patients with cerebellar cortical atrophy ${ }^{24}$ and other cerebellar degenerative diseases. ${ }^{44}$ Saiz et $a l^{16}$ recently identified GAD-Ab in the serum of three patients with late onset diabetes mellitus, cerebellar ataxia/degeneration, and increased titres of antithyroid antibodies, particularly TPO-Ab. The immunological and clinical profiles of these patients are strikingly similar to those in our current series, although in the two patients we studied a normal GAD titre was found in one patient and a borderline increased titre in the other. Finally, ataxia associated with Hashimoto's thyroiditis might be the result of an autoimmune mediated calcium channelopathy. Disordered regulation of Purkinje cell voltage dependent calcium channels has been shown to lead to degeneration of Purkinje cells and ataxia, ${ }^{45}$ and $\mathrm{P} / \mathrm{Q}$-type voltage gated calcium channel antibodies have been detected in paraneoplastic disorders of the CNS. ${ }^{46}$ Indirect evidence from studies of thyrotoxic periodic paralysis, a calcium channel disease, and spinocerebellar ataxia points to a possible link between autoimmune thyroid disease and Purkinje cell-type calcium channelopathy. ${ }^{18} 3245$

The present report highlights the association of progressive non-familial adult onset cerebellar degeneration with Hashimoto's/ autoimmune thyroiditis, signalled by increased $\mathrm{TPO}-\mathrm{Ab}$ and evidence of more general autoimmunity. We recommend testing for antithyroid antibodies and other evidence of autoimmunity in patients who present with PNACD. Although our own therapeutic experience has been limited, we suggest that immune modulation therapies, such as plasmapheresis, IVIg, or immuno-suppressive agents, deserve a trial in these patients.

1 Mancall EL, McEntee WJ. Alterations of the cerebellar cortex in nutritional encephalopathy. Neurology 1965;15:303-

2 Victor M, Adams R, Mancall EL. A restricted form of cerebellar cortical degeneration occurring in alcoholic patients. Arch Neurol 1959;1:579-88.

3 Mason WP, Graus F, Lang B, et al. Small-cell lung cancer, paraneoplastic cerebellar degeneration and the Lambertparaneoplastic cerebellar degeneration and the Lambert-

4 Déjerine J, Thomas A. L'atrophie olivo-ponto-cerebelleuse. Nouvelle Icongraphy de la Salpétriére 1900;13:330.

5 Barnard R, Campbell M, McDonald W. Pathological findings in a case of hypothyroidism with ataxia. F Neurol Neurosurg Psychiatry 1971;34:755-60.

6 Bonuccelli U, Nuti A, Monzani F, et al. Familial occurrence of hypothyroidism and cerebellar ataxia. Funct Neurol1991; 6:171-5.

7 Cremer GM, Goldstein NP, Paris J. Myxedema and ataxia. Neurology 1969;19:37-46.

8 Erkulvrawatr S. Gait ataxia in hypothyroidism. $7 \mathrm{Ky} \mathrm{Med}$ Assoc 1977;75:581-2.

9 Gentilini M, Palmieri M. Myxedematous cerebellar ataxia: description of a case. Rivista di Patologica Nervosae Mentale description of a casc

10 Hammar CH, Regli F. Cerebellar ataxia due to hypothyroidism in adults. Dtsch Med Wochenschr 1975;100:1504-6.

11 Jellinek EH, Kelly RE. Cerebellar syndrome in myxoedema. Lancet 1960;2:225-7.

12 Söderbergh G. Encore un cas de myxoedeme avec symptomes cerebelleux. Rev Neurol (Paris) 1911;22:86-9.

3 Somalwar A, Jalgaonkar P. Bilateral cerebellar syndrome in a case of myxoedema. F Indian Med Assoc1996;94:241.

14 Takayanagi K, Satoth A, Yoshimura T, et al. A case of myxoedema associated with cerebellar ataxia and various neurological findings. Nippon Naika Gakkai Zasshi 1982;71:9958. 
15 Wiebel J. Cerebellar-ataxic syndrome in children and adolescents with hypothyroidism under treatment. Acto adolescents with hypothyroid.

16 Saiz A, Arpa J, Sagasta A, et al. Autoantibodies to glutamic acid decarboxylase in three patients with cerebellar ataxia, late-onset insulin-dependent diabetes mellitus, and polyendocrine autoimmunity. Neurology 1997;49:1026-30.

17 Chiu A, Sherman S. Clinical manifestations and differential diagnosis of hypothyroidism. In: Falk SA, ed. Thyroid disease: endocrinology, surgery, nuclear medicine, and radiotherapy. 2nd ed. Philadelphia: Lippincott-Raven, 1997 $379-1$

18 Adams R, Victor M, Ropper A. Principles of neurology. 6th ed. New York: McGraw-Hill, 1997:1084-6.

19 Dalmau JO, Posner JB. Paraneoplastic syndromes. Arch Neurol 1999;56:405-8.

20 Dropcho EJ. Autoimmune central nervous system paraneoplastic disorders: mechanisms, diagnosis, and therapeutic plastic disorders: mechanisms, diagnosis, and

21 Porta-Etessam J, Berbel A, Martinez-Salio A, et al. Paraneoplastic cerebellar degeneration with symmetrical panplastic cerebellar degeneration with symmetrical

22 Roy M, Stip E, Black D, et al. Cerebellar degeneration following acute lithium intoxication. Rev Neurol (Paris) 1998;154:546-8.

23 Holmes GM. A form of familial degeneration of the cerebellum. Brain 1907;30:466

24 Honnorat J, Trouillas P, Thivolet C, et al. Autoantibodies to glutamate decarboxylase in a patient with cerebellar cortical atrophy, peripheral neuropathy, and slow eye movements. Arch Neurol 1995; 52:462-8.

25 Tanaka M, Kawarabayashi T, Okamoto K, et al. Reduction of cerebral blood flow and metabolic rate of oxygen in case of hypothyroidism presenting with cerebellar ataxia. Rinsho shinkeigaku 1987;27:1262-5.

26 Dayan CM, Daniels GH. Chronic autoimmune thyroiditis. N Eng F Med 1996;335:99-107.

27 Wyngaarden J, Lloyd S. Cecil textbook of medicine. 17th ed. Philadelphia: WB Saunders, 1985:1275-99.

28 Dropcho EJ. Neurologic paraneoplastic syndromes. F Neurol Sci 1998;153:264-78.

29 Manto M, Rondeaux P, Jacquy J, et al. Subacute pancerebellar syndrome associated with systemic lupus erythromatosus. Clin Neurol Neurosurg 1996;98:157-60.

30 Price TR, Netsky MG. Myxedema and ataxia; cerebella alterations and neural myxedema bodies. Neurology 1966 16:957-62.

31 Uyematsu S. A case of myxedematous psychosis: clinical and pathological report. Arch Neurol Psychiatry 1920;3 252-76.

32 Pinchera A, Fenzi G, Chiovato L, et al. Autoimmune hypothyroidism. In: Drexhage HA, Wiersinga WM, eds. The thyroid and autoimmunity. New York: Elsevier, 1986;73-8.
33 Becker KL, Ferguson RH, McConahey WM. The connective tissue diseases and symptoms associated with Hashimoto's thyroiditis. N Engl f Med 1963;268:277-80.

34 Pryse-Phillips W. The epidemiology of multiple sclerosis. In: Cook SD, ed. Handbook of multiple sclerosis, 2nd ed. New York: M Dekker, 1996:1-17.

35 Bednarczuk T, Kennerdell J, Wall J. Thyroid-associated ophthalmopathy: etiology and pathogenesis. In: Falk SA, ed. Thyroid disease: endocrinology, surgery, nuclear medicine, and radiotherapy. 2nd ed. Philadelphia: Lippincott-Raven, 1997:341-57.

36 Cohen L, Mouly S, Tassan P, et al. A woman with a relapsing psychosis who got better with prednisone. Lancet 1996; 347:1228

37 Concetta M. Forchetti, G. Katsamakis, et al. Autoimmune thyroiditis and a rapidly progressive dementia: global hypoperfusion on SPECT scanning suggests a possible mechanism. Neurology 1997;49:623-6.

38 Shaw PJ, Walls TJ, Newman PK, et al. Hashimoto's encephalopathy: a steroid-responsive disorder associated with high anti-thyroid antibody titers: report of five cases. Neurology 1991;41:228-33.

39 Shein M, Apter A, Dickerman Z, et al. Encephalopathy in compensated Hashimoto's thyroiditis: a clinical expression of autoimmune cerebral vasculitis. Brain Dev 1986;8:60-4.

40 Nickel SN, Frame B. Neurologic manifestations of myxedema. Neurology 1958;8:511-17.

41 McGeer PL, McGeer EG. Amino acid neurotransmitters. In: Siegel G, Agranoff B, Albers RW, eds. Basic neurochemistry: molecular, cellular, and medical aspects. 4th ed. New York: Raven, 1989:311-32.

42 Gilman S, Bloedel J, Lechtenberg R. Disorders of the cerebelum. Philadelphia: FA Davis, 1981:244-50.

43 Grimaldi L, Quattrini A, Martino G. Cerebellar autoantibodies in patients with isolated or associated stiff-man syndrome and cerebellar atrophies. Neurology 1990;40(suppl 1):117-18.

44 Nemni R, Bonifacio E, Mortara A, et al. Characterization and quantification of autoantibodies to glutamic acid decarboxylase in neurological syndromes. F. Neurol 1996; 243(suppl 2):S23.

45 Zuchenko O, Bailey J, Bonnen P, et al. Autosomal dominant cerebellar ataxia (SCA6) associated with small polyglutamine expansions in the $\alpha$ 1A-voltage-dependent calcium channel. Nature Genetics 1997;15:62-9.

46 Voltz R, Carpentier AF, Rosenfield MR, et al. P/Q-type voltage-gated calcium channel antibodies in paraneoplastic disorders of the central nervous system. Muscle Nerve 1999;22:119-22. 